Journal of Organometallic Chemistry, 387 (1990) 83-94

Elsevier Sequoia S.A., Lausanne - Printed in The Netherlands

JOM 20657

\title{
Structural characterization of tripodal phosphine complexes of transition metals. Examples of chair and twisted-boat conformations in six-membered chelate rings
}

\author{
Shiuh-Tzung Liu *, Gwo-Jiun Liu, Chien-Hung Yieh, Ming-Chu Cheng \\ and Shie-Ming Peng
}

Department of Chemistry, National Taiwan University, Taipei 10764 (Taiwan)

(Received November 11th, 1989)

\begin{abstract}
Complexation of a "hybrid" tripodal ligand 2,2-bis(diphenylphosphinomethyl)1-methoxypropane $\left(\mathbf{P}_{2} \mathbf{O}\right)$ with group VI carbonyls, nickel(II) and palladium(II) compounds has been studied. Thus $\mathrm{P}_{2} \mathrm{O}$ reacts with $\mathrm{M}(\mathrm{CO})_{6}, \mathrm{NiCl}_{2}$ and $\mathrm{PdCl}_{2}$ to give $\left(\mathbf{P}_{2} \mathbf{O}\right) \mathbf{M}(\mathrm{CO})_{4}(\mathbf{M}=\mathrm{Cr}, \mathbf{M o}, \mathbf{W}),\left(\mathbf{P}_{2} \mathbf{O}\right) \mathrm{NiCl}_{2}$ and $\left(\mathbf{P}_{2} \mathbf{O}\right) \mathrm{PdCl}_{2}$, respectively. All the complexes have been characterized by spectroscopy and elemental analysis, and the crystal structures of the complexes of $\left(\mathbf{P}_{2} \mathrm{O}\right) \mathrm{Mo}(\mathrm{CO})_{4}(\mathbf{4 b}),\left(\mathrm{P}_{2} \mathrm{O}\right) \mathrm{W}(\mathrm{CO})_{4}(4 \mathrm{c})$ and $\left(\mathbf{P}_{2} \mathbf{O}\right) \mathrm{NiCl}_{2}(6)$ have been determined by X-ray diffraction. In all cases, the tripodal ligand behaves solely as a bidentate with two phosphorus donors coordinating to the metal centers. In the examination of the conformations of the six-membered chelate rings, it was found that both $4 \mathrm{~b}$ and $4 \mathrm{c}$ were in the twisted-boat form; whereas 6 was in the chair form. The possible reasons for this conformational difference are discussed.
\end{abstract}

\section{Introduction}

Tripodal ligands have received much attention [1-8], but such systems have been limited to the same donor atoms, such as all-phosphorus donors in triphos 1, and a few nitrogen and oxygen containing "mixed" ligands (2) have been used for complexation with titanium metal [8]. Owing to the tripod-like geometry of these ligands, they can ligate three dimensionally such as in $f a c$-(triphos) $\mathrm{Cr}(\mathrm{CO})_{3}$ [3]. This characteristic feature would make tripod-like ligands containing different ligating sites useful for coordination studies. We recently developed the synthesis of a new tripodal ligand 3, which has both "hard" and "soft" donor sites [6]. Here, we present our results on the coordinating behavior of 3 toward transition metals.

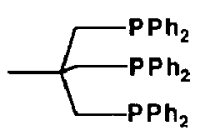

(1)

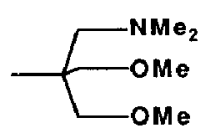

(2)

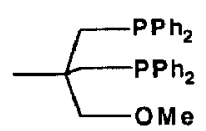

(3) 


\section{Results and discussion}

\section{Group VI complexes}

The thermal reaction of 3 with $\mathrm{Cr}(\mathrm{CO})_{6}$ in boiling xylene was found to give $(3) \mathrm{Cr}(\mathrm{CO})_{4}$ in $53 \%$ yield (eq. 1). Similarly, when $\mathrm{Mo}(\mathrm{CO})_{6}$ or $\mathrm{W}(\mathrm{CO})_{6}$ are heated with 3 in toluene the corresponding (3) $\mathrm{M}(\mathrm{CO})_{4}$ complexes (eq. 1) are obtained. All the reactions were monitored as a function of time by IR spectroscopy, which showed that $8-10 \mathrm{~h}$ were required for all of the metal carbonyl to react with 3 . Prolonged heating or photochemical activation of $\mathbf{4 b}$ degrades the tetracarbonyl species with no indication of formation of the tricarbonyl species, $(3) \mathrm{M}(\mathrm{CO})_{3}$. Another route to the tricarbonyl species involves the reaction of $\mathrm{M}\left(\mathrm{CH}_{3} \mathrm{CN}\right)_{3}(\mathrm{CO})_{3}$ or (cycloheptatriene) $\mathrm{M}(\mathrm{CO})_{3}$ with 3 to give 4 . Clearly a "hard" donor atom cannot be forced to coordinate to a group VI metal center, not even through a tripodal linkage. In Table 1 are listed the spectroscopic and analytical data for $\mathbf{4 a - 4 c}$. The methylene units attached to the phosphorus group are divided into two groups: those with hydrogen atoms cis to the methoxymethyl group in the chelate ring, and those in which they are trans. Consequently, the ${ }^{1} \mathrm{H}$ NMR spectra of $4 \mathbf{a}-\mathbf{4 c}$ showed an absorption pattern of $\mathrm{ABX}$ for these protons because of the geminal coupling $(J(\mathrm{H}-\mathrm{C}-\mathrm{H}))$ and the splitting by phosphorus $(J(\mathrm{P}-\mathrm{C}-\mathrm{H}))$. The ${ }^{31} \mathrm{P}$ NMR coordi-

Table 1

Spectroscopic and analytical data for $\mathbf{4 a - 4 c}$

\begin{tabular}{|c|c|c|c|c|c|c|c|}
\hline \multirow[t]{3}{*}{ Complex } & \multirow[t]{3}{*}{${ }^{1}$ H NMR } & \multirow[t]{3}{*}{${ }^{31} \mathrm{P} \mathrm{NMR}^{a}$} & \multirow[t]{3}{*}{ IR (Toluene) } & \multicolumn{4}{|c|}{ Analysis $(\%)$} \\
\hline & & & & \multicolumn{2}{|l|}{ Calcd } & \multicolumn{2}{|l|}{ Found } \\
\hline & & & & $\overline{\mathrm{C}}:$ & $\mathrm{H}:$ & $\overline{\mathrm{C}:}$ & $\mathrm{H}:$ \\
\hline$\overline{4 a}$ & $\begin{array}{l}7.62-7.52(\mathrm{~m}, 4 \mathrm{H}) \\
7.52-7.45(\mathrm{~m}, 4 \mathrm{H}) \\
7.45-7.30(\mathrm{~m}, 12 \mathrm{H}) \\
2.94(\mathrm{~s}, 3 \mathrm{H}), 2.76(\mathrm{~s}, 2 \mathrm{H}) \\
2.52(\mathrm{dd}, J 15,6 \mathrm{~Hz}, 2 \mathrm{H}) \\
2.30(\mathrm{dd}, J 15,9 \mathrm{~Hz}, 2 \mathrm{H}) \\
0.75(\mathrm{~s}, 3 \mathrm{H})\end{array}$ & $41.09(66.2)$ & $\begin{array}{l}2006(\mathrm{~m}) \\
1999(\mathrm{~m}) \\
1917(\mathrm{~m}) \\
1882(\mathrm{~s})\end{array}$ & $\begin{array}{l}\mathrm{C}_{34} \mathrm{H}_{32} \\
64.35\end{array}$ & $\begin{array}{c}\mathrm{O}_{5} \mathrm{P}_{2} \mathrm{Cr} \\
5.08\end{array}$ & 63.15 & 5.15 \\
\hline $4 b$ & $\begin{array}{l}7.68-7.53(\mathrm{~m}, 4 \mathrm{H}) \\
7.53-7.42(\mathrm{~m}, 4 \mathrm{H}) \\
7.42-7.30(\mathrm{~m}, 12 \mathrm{H}) \\
2.99(\mathrm{~s}, 3 \mathrm{H}), 2.83(\mathrm{~s}, 2 \mathrm{H}) \\
2.53(\mathrm{dd}, J 15,6 \mathrm{~Hz}, 2 \mathrm{H}) \\
2.42(\mathrm{dd}, J 15,9 \mathrm{~Hz}, 2 \mathrm{H}) \\
0.75(\mathrm{~s}, 3 \mathrm{H})\end{array}$ & $19.59(40.7)$ & $\begin{array}{l}2017(\mathrm{~m}) \\
1919(\mathrm{~m}) \\
1892(\mathrm{~s})\end{array}$ & $\begin{array}{l}\mathrm{C}_{34} \mathrm{H}_{32} \\
60.18\end{array}$ & $\begin{array}{c}\mathrm{O}_{5} \mathrm{P}_{2} \mathrm{Mo} \\
4.75\end{array}$ & 60.09 & 4.76 \\
\hline $4 c$ & $\begin{array}{l}7.62-7.50(\mathrm{~m}, 4 \mathrm{H}) \\
7.50-7.42(\mathrm{~m}, 4 \mathrm{H}) \\
7.42-7.39(\mathrm{~m}, 12 \mathrm{H}) \\
2.98(\mathrm{~s}, 3 \mathrm{H}), 2.82(\mathrm{~s}, 2 \mathrm{H}) \\
2.63(\mathrm{dd}, J 15,6 \mathrm{~Hz}, 2 \mathrm{H}) \\
2.50(\mathrm{dd}, J 15,9 \mathrm{~Hz}, 2 \mathrm{H}) \\
0.76(\mathrm{~s}, 3 \mathrm{H})\end{array}$ & $0.01(25.1)$ & $\begin{array}{l}2014(\mathrm{~m}) \\
1913(\mathrm{~m}) \\
1882(\mathrm{~s})\end{array}$ & $\begin{array}{l}\mathrm{C}_{34} \mathrm{H}_{32} \\
53.28\end{array}$ & $\begin{array}{c}\mathrm{O}_{5} \mathrm{P}_{2} \mathrm{~W} \\
4.21\end{array}$ & 53.24 & 4.29 \\
\hline
\end{tabular}

\footnotetext{
${ }^{a}$ The coordination chemical shifts $\left(\Delta_{\delta}\right)$ are given in the parentheses. $\Delta_{\delta}=\delta$ (complex) $-\delta$ (free ligand) in ppm.
} 


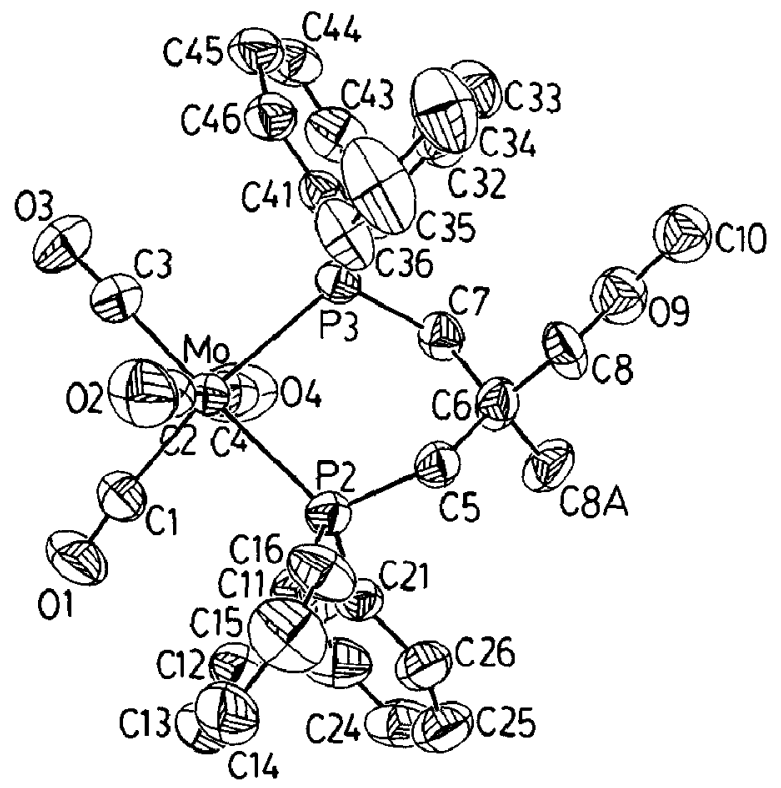

Fig. 1. The ORTEP plot of complex of $\mathbf{4 b}$.

nation chemical shifts (Table 1 ) which are found to fall in the order $\mathrm{Cr}>\mathrm{Mo}>\mathrm{W}$, are similar to those for (dppe) $\mathrm{M}(\mathrm{CO})_{4}$ [dppp = 1,3-bis(diphenylphosphino)propane] [9] and $\left(\mathbf{P}_{2} \mathrm{~S}\right) \mathrm{M}(\mathrm{CO})_{4}(5)[7]$.

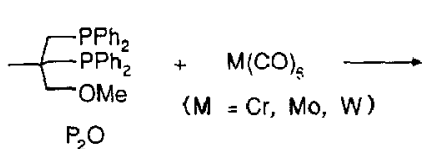

$\mathrm{P}_{2} \mathrm{O}$

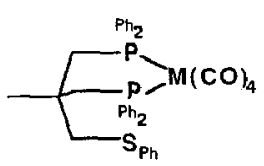

(5)

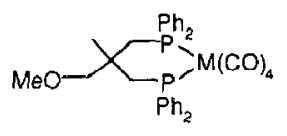

Crystals suitable for the X-ray study of $\mathbf{4 b}$ and $\mathbf{4 c}$ were obtained from dichloromethane and methanol. Complexes $\mathbf{4 b}$ and $\mathbf{4 c}$ are isomorphorus, i.e. they have the same atomic residues at crystallographic sites. Both molecules contain tripodal ligand 3 coordinated to the $\mathrm{M}(\mathrm{CO})_{4}$ moiety through two phosphorus atoms to form a cis octahedral geometry. Figures 1 and 2 show different views of $\mathbf{4 b}$. The atomic coordinates and thermal parameters for $\mathbf{4 b}$ and $\mathbf{4 c}$ are given in Table 2 and Table 3 , respectively. Selected bond distances and bond angles are listed in Table 4 . All bond distances are within expected ranges, such as 2.50-2.52 $\AA$ typical for metal-phosphorus bond lengths. In the structure of $\mathbf{4 b}$, the four metal carbonyl distances fall into two different groups whose average values are 1.983 and $1.998 \AA$. The two 


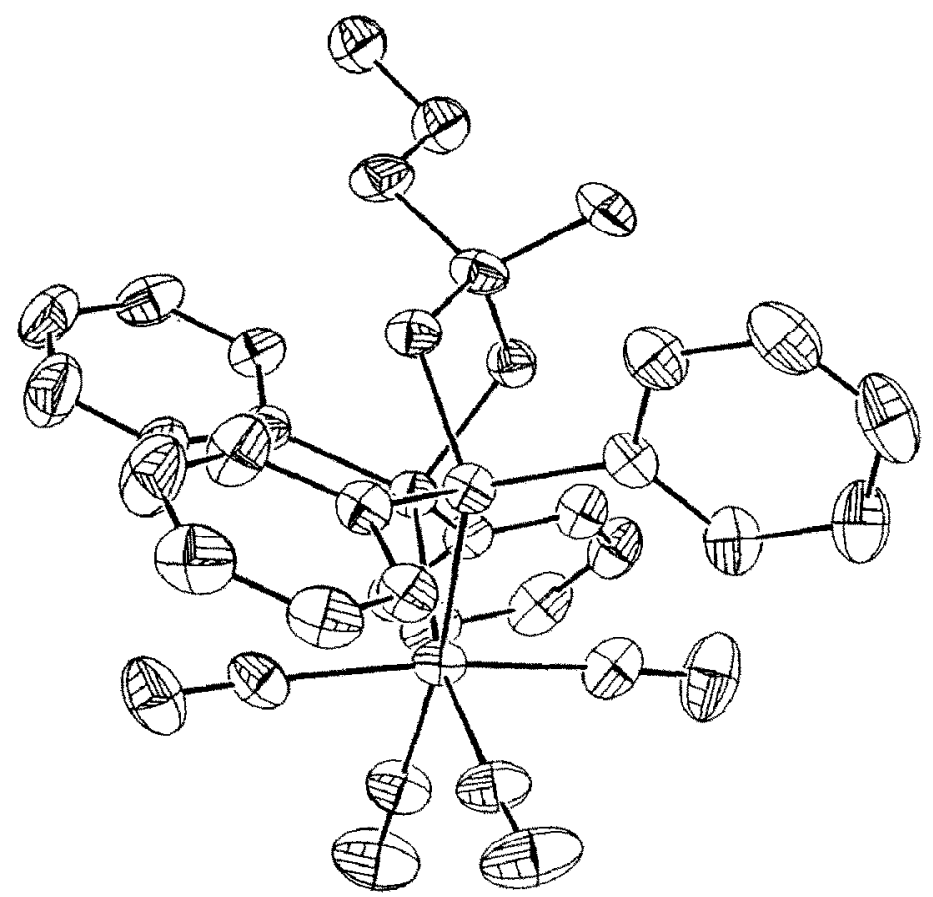

Fig. 2. Another view of the crystal structure of $\mathbf{4 b}$.

metal-carbon bonds trans to phosphorus atoms are slightly shorter, as expected, than those mutually trans to carbonyl groups; but the inverse is observed in $\mathbf{4 c}$, 1.984 vs. $1.970 \AA$.

$\mathrm{NiCl}_{2}$ and $\mathrm{PdCl}_{2}$ complexes

A solution of 3 in acetone was treated with $\mathrm{NiCl}_{2} \cdot \mathrm{DME}$ [DME $=1,2-$ dimethoxyethane] (also in acetone) at room temperature, complex 6 was immediately formed as was indicated by the change in color to orange. However, when this solution was heated under reflux for $2 \mathrm{~h}$, the complex could be isolated as an orange solid. The conductivity $\left(1.7 \Omega^{-1} \mathrm{~cm}^{2} \mathrm{~mol}^{-1}\right)$ and the electronic absorptions $(466,312,283,276$ and $239 \mathrm{~nm})$ are almost identical with those reported for the complex (triphos) $\mathrm{NiCl}_{2}$ [5].

The complex $\left(\mathbf{P}_{2} \mathbf{O}\right) \mathrm{NiCl}_{2} \cdot \mathrm{CH}_{2} \mathrm{Cl}_{2}$ can be isolated as a crystalline solid; its structure is depicted in Figs. 3 and 4. The atomic coordinates and thermal parameters are listed in Table 5. Some important bond distances and angles of the complex 6 and those of $\mathbf{4 b}$ and $\mathbf{4 c}$ are listed in Table 4 for comparison. The Ni(II) center is tetra-coordinated with two chloride ligands and two phosphorus donors in a square-planar geometry, with the $\mathrm{Ni}$ atom slightly $(0.031(1) \AA)$ out of the plane. The oxygen donor of the tripodal ligand 3 remains uncoordinated, which is similar to that in the complex (triphos) $\mathrm{NiCl}_{2}$ that has one dangling phosphorus donor [10]. A symmetry plane passes through the complex at the $\mathrm{Ni}$ atom, the $\mathrm{C} 5$ carbon and the oxygen atom. The $\mathrm{Ni}-\mathrm{P}$ bond distances are $2.164(2) \AA$, and the $\mathrm{Ni}-\mathrm{Cl}$ are $2.207(2)$ $\AA$. The angles of both $\mathrm{Cl}-\mathrm{Ni}-\mathrm{Cl}$ and $\mathrm{P}-\mathrm{Ni}-\mathrm{P}$ are slightly distorted from $90^{\circ}$. 
Table 2

The atomic coordinates and thermal parameters for $\mathbf{4 b}$

\begin{tabular}{|c|c|c|c|c|}
\hline & $x$ & $y$ & $z$ & $B_{\text {iso }}$ \\
\hline Mo & $0.72723(4)$ & $0.35416(2)$ & $0.25734(2)$ & $3.63(2)$ \\
\hline $\mathbf{P} 2$ & $0.86855(11)$ & $0.25030(7)$ & $0.23008(7)$ & $3.60(5)$ \\
\hline P3 & $0.73187(11)$ & $0.28676(7)$ & $0.39267(7)$ & $3.53(6)$ \\
\hline $\mathrm{C} 1$ & $0.7576(5)$ & $0.4078(3)$ & $0.1575(3)$ & $5.4(3)$ \\
\hline 01 & $0.7817(4)$ & $0.43747(21)$ & $0.10157(23)$ & $8.8(3)$ \\
\hline $\mathrm{C} 2$ & $0.5954(4)$ & $0.2956(3)$ & $0.1842(3)$ & $4.7(3)$ \\
\hline $\mathrm{O} 2$ & $0.5189(3)$ & $0.26523(23)$ & $0.14020(20)$ & $7.38(23)$ \\
\hline $\mathrm{C} 3$ & $0.6023(4)$ & $0.4277(3)$ & $0.2768(3)$ & $5.4(3)$ \\
\hline $\mathrm{O} 3$ & $0.5296(4)$ & $0.47049(22)$ & $0.28669(23)$ & $8.24(25)$ \\
\hline $\mathrm{C4}$ & $0.8473(5)$ & $0.4231(3)$ & $0.3238(3)$ & $5.2(3)$ \\
\hline 04 & $0.9138(4)$ & $0.46626(22)$ & $0.36050(24)$ & $8.35(25)$ \\
\hline $\mathrm{C} 5$ & $0.8736(4)$ & $0.16613(24)$ & $0.2969(3)$ & $3.88(22)$ \\
\hline $\mathrm{C} 6$ & $0.9226(4)$ & $0.1722(3)$ & $0.3914(3)$ & $4.18(22)$ \\
\hline $\mathrm{C} 7$ & $0.8812(4)$ & $0.24495(25)$ & $0.43072(24)$ & $3.94(23)$ \\
\hline $\mathrm{C} 11$ & $0.8201(4)$ & $0.20571(25)$ & $0.1287(3)$ & $3.87(21)$ \\
\hline $\mathrm{C} 12$ & $0.8478(4)$ & $0.2419(3)$ & $0.0591(3)$ & $4.48(24)$ \\
\hline $\mathrm{C} 13$ & $0.8091(5)$ & $0.2123(3)$ & $-0.0189(3)$ & $5.3(3)$ \\
\hline $\mathrm{C} 14$ & $0.7415(5)$ & $0.1483(3)$ & $-0.0306(3)$ & $6.3(3)$ \\
\hline $\mathrm{C} 15$ & $0.7122(5)$ & $0.1120(3)$ & $0.0362(3)$ & $7.2(3)$ \\
\hline C16 & $0.7509(5)$ & $0.1405(3)$ & $0.1163(3)$ & $5.8(3)$ \\
\hline $\mathrm{C} 21$ & $1.0242(4)$ & $0.2731(3)$ & $0.2264(2)$ & $3.9(2)$ \\
\hline $\mathrm{C} 22$ & $1.0641(4)$ & $0.3474(3)$ & $0.2360(3)$ & $5.0(3)$ \\
\hline $\mathrm{C} 23$ & $1.1825(5)$ & $0.3663(3)$ & $0.2332(3)$ & $6.5(3)$ \\
\hline $\mathrm{C} 24$ & $1.2616(5)$ & $0.3100(4)$ & $0.2216(3)$ & $7.1(4)$ \\
\hline $\mathrm{C} 25$ & $1.2217(5)$ & $0.2372(3)$ & $0.2098(3)$ & $6.6(3)$ \\
\hline $\mathrm{C} 26$ & $1.1049(4)$ & $0.2181(3)$ & $0.2129(3)$ & $5.4(3)$ \\
\hline C31 & $0.6270(4)$ & $0.2112(3)$ & $0.4067(3)$ & $3.9(2)$ \\
\hline $\mathrm{C} 32$ & $0.6221(4)$ & $0.1831(3)$ & $0.4853(3)$ & $4.6(3)$ \\
\hline C33 & $0.5485(5)$ & $0.1229(3)$ & $0.4947(3)$ & $6.1(3)$ \\
\hline C34 & $0.4785(6)$ & $0.0897(3)$ & $0.4282(4)$ & $8.1(4)$ \\
\hline C35 & $0.4828(6)$ & $0.1171(3)$ & $0.3504(3)$ & 9.1(4) \\
\hline $\mathrm{C} 36$ & $0.5556(5)$ & $0.1771(3)$ & $0.3409(3)$ & $5.9(3)$ \\
\hline C41 & $0.7153(4)$ & $0.3529(2)$ & $0.4763(2)$ & $3.7(2)$ \\
\hline $\mathrm{C} 42$ & $0.8130(4)$ & $0.3844(3)$ & $0.5288(3)$ & $4.7(3)$ \\
\hline $\mathrm{C} 43$ & $0.7949(5)$ & $0.4362(3)$ & $0.5896(3)$ & $5.7(3)$ \\
\hline C44 & $0.6807(5)$ & $0.4548(3)$ & $0.5979(3)$ & $6.2(3)$ \\
\hline $\mathrm{C} 45$ & $0.5841(5)$ & $0.4233(3)$ & $0.5459(3)$ & $5.3(3)$ \\
\hline $\mathrm{C} 46$ & $0.6015(4)$ & $0.3734(3)$ & $0.4852(3)$ & $4.5(2)$ \\
\hline $\mathrm{C} 8$ & $0.8728(5)$ & $0.0993(3)$ & $0.4270(3)$ & $4.8(3)$ \\
\hline $\mathrm{C} 8 \mathrm{~A}$ & $1.0620(4)$ & $0.1741(3)$ & $0.4098(3)$ & $5.2(3)$ \\
\hline O9 & $0.9177(4)$ & $0.1043(3)$ & $0.5148(3)$ & $5.5(1)$ \\
\hline $\mathrm{C} 10$ & $0.8694(6)$ & $0.0423(4)$ & $0.5580(4)$ & $5.6(2)$ \\
\hline O9A & $1.0898(10)$ & $0.1036(6)$ & $0.3727(7)$ & $6.0(3)$ \\
\hline C10A & $1.1980(21)$ & $0.0781(13)$ & $0.3912(14)$ & $10.3(7)$ \\
\hline
\end{tabular}

Displacement reactions of 6 by other phosphines were also studied. $\left(\mathbf{P}_{\mathbf{2}} \mathbf{O}\right) \mathrm{NiCl}_{2}$ undergoes no ligand substitution reactions with triphenylphosphine, even at elevated temperatures. On the other hand, dppe [dppe $=1,2$-bis(diphenylphosphino)ethane] reacts instantly with 6 to generate the free tripodal ligand 3 , (dppe) $\mathrm{NiCl}_{2}$ and (dppe) ${ }_{2} \mathrm{NiCl}_{2}$ as identified by ${ }^{31} \mathrm{P}$ NMR spectroscopy. 
Table 3

The atomic coordinates and thermal parameters for $\mathbf{4 c}$

\begin{tabular}{|c|c|c|c|c|}
\hline & $x$ & $y$ & $z$ & $B_{\text {iso }}$ \\
\hline W & $0.72733(3)$ & $0.354186(17)$ & $0.257170(18)$ & $3.400(14)$ \\
\hline $\mathrm{P} 2$ & $0.86782(17)$ & $0.25007(11)$ & $0.22972(11)$ & $3.38(9)$ \\
\hline $\mathrm{P} 3$ & $0.73236(16)$ & $0.28701(11)$ & $0.39226(11)$ & $3.26(9)$ \\
\hline $\mathrm{C} 1$ & $0.7596(7)$ & $0.4076(4)$ & $0.1567(5)$ & $5.0(4)$ \\
\hline O1 & $0.7827(6)$ & $0.4372(3)$ & $0.1003(4)$ & $8.2(4)$ \\
\hline $\mathrm{C} 2$ & $0.5961(7)$ & $0.2975(5)$ & $0.1849(4)$ & $4.6(5)$ \\
\hline $\mathrm{O} 2$ & $0.5188(5)$ & $0.2657(4)$ & $0.1402(3)$ & $6.9(4)$ \\
\hline $\mathrm{C} 3$ & $0.6030(7)$ & $0.4281(4)$ & $0.2766(5)$ & $5.0(4)$ \\
\hline O3 & $0.5298(5)$ & $0.4713(3)$ & $0.2876(4)$ & $7.9(4)$ \\
\hline $\mathrm{C4}$ & $0.8465(7)$ & $0.4219(4)$ & $0.3237(5)$ & $4.9(5)$ \\
\hline $\mathrm{O} 4$ & $0.9148(5)$ & $0.4656(4)$ & $0.3610(4)$ & $7.9(4)$ \\
\hline $\mathrm{C} 5$ & $0.8737(6)$ & $0.1665(4)$ & $0.2962(4)$ & $3.7(3)$ \\
\hline C6 & $0.9226(6)$ & $0.1711(4)$ & $0.3911(4)$ & $3.8(3)$ \\
\hline $\mathrm{C} 7$ & $0.8816(6)$ & $0.2444(4)$ & $0.4304(4)$ & $3.7(4)$ \\
\hline C11 & $0.8188(6)$ & $0.2056(4)$ & $0.1283(4)$ & $3.5(3)$ \\
\hline $\mathrm{C} 12$ & $0.8479(6)$ & $0.2415(4)$ & $0.0595(5)$ & $4.2(4)$ \\
\hline $\mathrm{C} 13$ & $0.8095(8)$ & $0.2123(5)$ & $-0.0197(5)$ & $5.2(5)$ \\
\hline $\mathrm{C} 14$ & $0.7409(8)$ & $0.1487(6)$ & $-0.0308(5)$ & $6.0(5)$ \\
\hline C15 & $0.7118(8)$ & $0.1118(5)$ & $0.0361(6)$ & $6.9(6)$ \\
\hline $\mathrm{C} 16$ & $0.7487(7)$ & $0.1404(5)$ & $0.1158(4)$ & $5.5(5)$ \\
\hline $\mathrm{C} 21$ & $1.0233(6)$ & $0.2732(4)$ & $0.2259(4)$ & $3.6(4)$ \\
\hline $\mathrm{C} 22$ & $1.0626(6)$ & $0.3481(5)$ & $0.2345(4)$ & $4.5(4)$ \\
\hline $\mathrm{C} 23$ & $1.1817(8)$ & $0.3666(5)$ & $0.2321(5)$ & $6.4(5)$ \\
\hline $\mathrm{C} 24$ & $1.2606(8)$ & $0.3107(7)$ & $0.2196(5)$ & $6.9(6)$ \\
\hline C25 & $1.2204(8)$ & $0.2366(6)$ & $0.2097(5)$ & $6.5(5)$ \\
\hline $\mathrm{C} 26$ & $1.1032(7)$ & $0.2176(4)$ & $0.2121(5)$ & $4.9(4)$ \\
\hline C31 & $0.6274(6)$ & $0.2111(4)$ & $0.4058(4)$ & $3.6(4)$ \\
\hline $\mathrm{C} 32$ & $0.6225(7)$ & $0.1837(4)$ & $0.4843(4)$ & $4.4(4)$ \\
\hline C33 & $0.5505(9)$ & $0.1233(5)$ & $0.4955(5)$ & $5.8(5)$ \\
\hline C34 & $0.4788(9)$ & $0.0892(5)$ & $0.4291(7)$ & $7.9(6)$ \\
\hline C 35 & $0.4830(9)$ & $0.1164(6)$ & $0.3502(6)$ & $8.7(6)$ \\
\hline C36 & $0.5572(8)$ & $0.1771(5)$ & $0.3397(5)$ & $5.8(5)$ \\
\hline $\mathrm{C} 41$ & $0.7139(6)$ & $0.3529(4)$ & $0.4758(4)$ & $3.4(3)$ \\
\hline $\mathrm{C} 42$ & $0.8124(6)$ & $0.3845(4)$ & $0.5291(4)$ & $4.3(4)$ \\
\hline $\mathrm{C} 43$ & $0.7919(8)$ & $0.4367(5)$ & $0.5903(5)$ & $5.6(5)$ \\
\hline $\mathrm{C} 44$ & $0.6779(9)$ & $0.4543(5)$ & $0.5982(5)$ & $5.8(5)$ \\
\hline $\mathrm{C} 45$ & $0.5831(7)$ & $0.4241(4)$ & $0.5459(5)$ & $4.9(5)$ \\
\hline $\mathrm{C} 46$ & $0.5996(7)$ & $0.3733(4)$ & $0.4846(4)$ & $4.2(4)$ \\
\hline $\mathrm{C} 8$ & $0.8731(7)$ & $0.0983(4)$ & $0.4277(4)$ & $4.3(4)$ \\
\hline $\mathrm{C} 8 \mathrm{~A}$ & $1.0628(6)$ & $0.1727(4)$ & $0.4097(4)$ & $4.6(4)$ \\
\hline $\mathrm{O} 9$ & $0.9167(6)$ & $0.1028(4)$ & $0.5144(4)$ & $5.54(17)$ \\
\hline $\mathrm{C} 10$ & $0.8719(9)$ & $0.0414(6)$ & $0.5597(6)$ & $5.3(3)$ \\
\hline O9A & $1.0900(17)$ & $0.1026(10)$ & $0.3728(10)$ & $6.0(4)$ \\
\hline C10A & $1.198(3)$ & $0.0809(19)$ & $0.3873(20)$ & $9.2(10)$ \\
\hline
\end{tabular}

The $\mathrm{Pd}^{\mathrm{II}}$ complex was formed by stirring a mixture of 3 and $\mathrm{PdCl}_{2}$ in dichloromethane and methanol, and was isolated as a yellow solid. The ${ }^{31} \mathrm{P}$ NMR showed a single absorption at $16.93 \mathrm{ppm}$, which indicates that two phosphorus donors coordinate to the metal center. The ${ }^{1} \mathrm{H}$ NMR chemical shift of the methylene unit attached to the oxygen atom is similar to that of $\mathbf{4 b}$, indicating that the oxygen donor remains uncoordinated. 
Table 4. Selected bond distances $(\AA)$ and bond angles $\left({ }^{\circ}\right)$ for $4 \mathbf{b}, 4 \mathbf{c}$ and 6 .

\begin{tabular}{|c|c|c|c|c|c|}
\hline $4 b$ & & $4 c$ & & 6 & \\
\hline Mo-P2 & $2.519(1)$ & W-P2 & $2.511(2)$ & $\mathrm{Ni}-\mathrm{Cl}$ & $2.207(2)$ \\
\hline Mo-P3 & $2.515(1)$ & W-P3 & $2.502(2)$ & $\mathrm{Ni}-\mathrm{P}$ & $2.164(2)$ \\
\hline Mo-C1 & $1.981(5)$ & W-C1 & $1.990(8)$ & $\mathrm{P} 2-\mathrm{C} 5$ & $1.826(4)$ \\
\hline $\mathrm{Mo}-\mathrm{C} 2$ & $2.012(5)$ & $\mathrm{W}-\mathrm{C} 2$ & $1.980(8)$ & $\mathrm{C} 5-\mathrm{C} 6$ & $1.537(5)$ \\
\hline Mo-C3 & $1.985(5)$ & $W-C 3$ & $1.979(8)$ & & \\
\hline Mo-C4 & $1.984(5)$ & W-C4 & $1.961(8)$ & & \\
\hline P2-C5 & $1.833(4)$ & P2-C5 & $1.815(7)$ & & \\
\hline $\mathrm{P} 3-\mathrm{C} 7$ & $1.842(5)$ & P3-C7 & $1.843(7)$ & & \\
\hline $\mathrm{C} 5-\mathrm{C} 6$ & $1.555(6)$ & C5-C6 & $1.555(9)$ & & \\
\hline $\mathrm{C} 6-\mathrm{C} 7$ & $1.541(6)$ & C6-C7 & $1.542(9)$ & & \\
\hline $\mathrm{C} 1-\mathrm{O} 1$ & $1.137(6)$ & $\mathrm{C} 1-\mathrm{O} 1$ & $1.135(9)$ & & \\
\hline $\mathrm{C} 2-\mathrm{O} 2$ & $1.150(6)$ & $\mathrm{C} 2-\mathrm{O} 2$ & $1.17(1)$ & & \\
\hline $\mathrm{C} 3-\mathrm{O} 3$ & $1.148(6)$ & $\mathrm{C} 3-\mathrm{O} 3$ & $1.159(9)$ & & \\
\hline $\mathrm{C} 4-\mathrm{O} 4$ & $1.154(6)$ & $\mathrm{C} 4-\mathrm{O} 4$ & $1.17(1)$ & & \\
\hline P2-Mo-P3 & $85.08(4)$ & P2-W-P3 & $85.10(6)$ & $\mathrm{Cl}-\mathrm{Ni}-\mathrm{Cl}^{\prime}$ & 92.24 \\
\hline P2-Mo-C1 & $88.6(1)$ & $\mathrm{P} 2-\mathrm{W}-\mathrm{C} 1$ & $88.0(2)$ & $\mathrm{Cl}-\mathrm{Ni}-\mathrm{P} 2$ & $86.11(7)$ \\
\hline $\mathrm{P} 2-\mathrm{Mo}-\mathrm{C} 2$ & $87.3(1)$ & $\mathrm{P} 2-\mathrm{W}-\mathrm{C} 2$ & $87.8(2)$ & $\mathrm{Cl}-\mathrm{Ni}-\mathrm{P} 2^{\prime}$ & $177.70(5)$ \\
\hline $\mathrm{P} 2-\mathrm{Mo}-\mathrm{C} 3$ & $173.9(2)$ & $\mathrm{P} 2-\mathrm{W}-\mathrm{C} 3$ & $174.0(2)$ & $\mathrm{P} 2-\mathrm{Ni}-\mathrm{P} 2^{\prime}$ & $85.49(7)$ \\
\hline P2-Mo-C4 & $98.2(2)$ & $\mathrm{P} 2-\mathrm{W}-\mathrm{C} 4$ & $98.2(2)$ & $\mathrm{Ni}-\mathrm{P} 2-\mathrm{C} 5$ & $120.1(1)$ \\
\hline P3-Mo-C1 & $169.0(2)$ & P3-W-C1 & $168.3(2)$ & $\mathrm{P} 2-\mathrm{C} 5-\mathrm{C} 6$ & $118.7(3)$ \\
\hline $\mathrm{P} 3-\mathrm{Mo}-\mathrm{C} 2$ & $100.4(1)$ & $\mathrm{P} 3-\mathrm{W}-\mathrm{C} 2$ & $100.7(2)$ & & \\
\hline P3-Mo-C3 & 93.1(1) & P3-W-C3 & $93.2(2)$ & & \\
\hline P3-Mo-C4 & $84.2(1)$ & P3-W-C4 & $83.7(2)$ & & \\
\hline $\mathrm{C} 2-\mathrm{Mo}-\mathrm{C} 4$ & $173.2(2)$ & $C 2-W-C 4$ & $172.9(3)$ & & \\
\hline Mo-P2-C5 & $114.8(1)$ & $\mathrm{W}-\mathrm{P} 2-\mathrm{C} 5$ & $115.0(2)$ & & \\
\hline Mo-P3-C7 & $110.5(1)$ & $\mathrm{W}-\mathrm{P} 3-\mathrm{C} 7$ & $110.7(2)$ & & \\
\hline $\mathrm{Mo}-\mathrm{C} 1-\mathrm{O} 1$ & $176.1(5)$ & $\mathrm{W}-\mathrm{Cl}-\mathrm{O} 1$ & $177.2(7)$ & & \\
\hline $\mathrm{Mo}-\mathrm{C} 2-\mathrm{O} 2$ & $176.8(4)$ & $\mathrm{W}-\mathrm{C} 2-\mathrm{O} 2$ & $177.7(6)$ & & \\
\hline $\mathrm{Mo}-\mathrm{C} 3-\mathrm{O} 3$ & $178.9(4)$ & $\mathrm{W}-\mathrm{C} 3-\mathrm{O} 3$ & $179.6(6)$ & & \\
\hline $\mathrm{Mo}-\mathrm{C} 4-\mathrm{O} 4$ & $176.5(4)$ & $\mathrm{W}-\mathrm{C} 4-\mathrm{O} 4$ & $176.4(7)$ & & \\
\hline
\end{tabular}

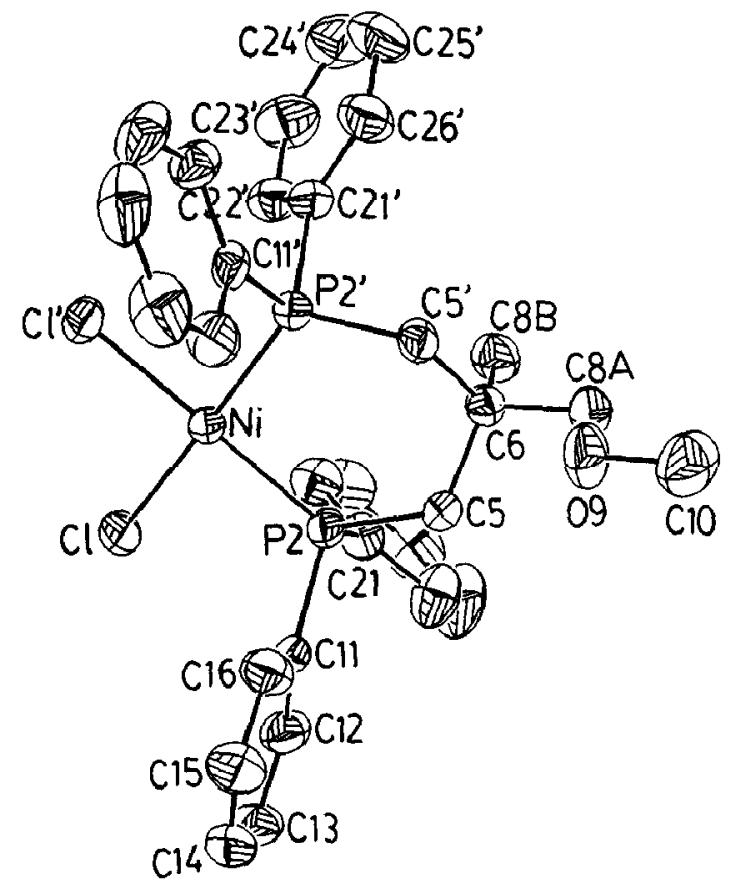

D: 2 The ORTFP nlot of complex of 6. 


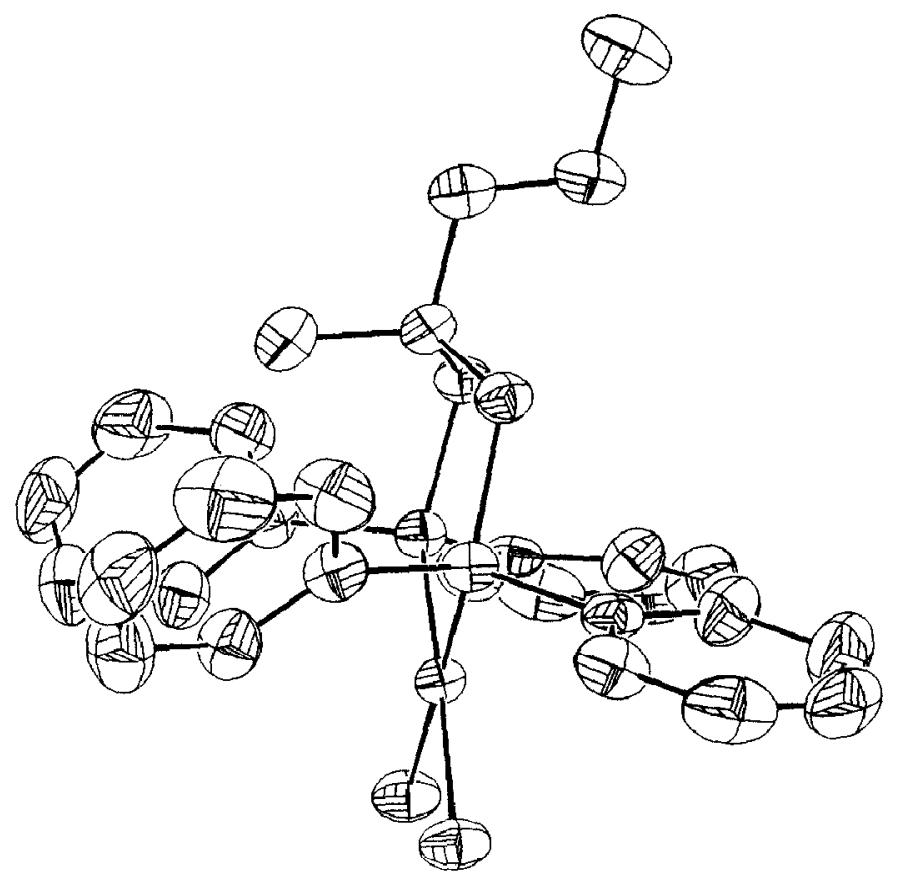

Fig. 4. Another view of the crystal structure of 6.

Tablc 5

The atomic coordinates and thermal parameters for 6

\begin{tabular}{lllll}
\hline & $x$ & $y$ & $z$ & $B_{\text {iso }}$ \\
\hline $\mathrm{Ni}$ & $0.68969(10)$ & $1 / 4$ & $0.27588(8)$ & $2.28(4)$ \\
$\mathrm{Cl}$ & $0.82647(14)$ & $0.33975(6)$ & $0.22496(12)$ & $3.45(6)$ \\
$\mathrm{P} 2$ & $0.55522(14)$ & $0.34038(6)$ & $0.31861(11)$ & $2.48(6)$ \\
$\mathrm{C} 5$ & $0.4184(5)$ & $0.32030(23)$ & $0.4044(4)$ & $2.69(21)$ \\
$\mathrm{C} 6$ & $0.3148(7)$ & $1 / 4$ & $0.3637(6)$ & $2.7(3)$ \\
$\mathrm{C} 8 \mathrm{~B}$ & $0.2193(8)$ & $1 / 4$ & $0.2117(6)$ & $3.9(4)$ \\
$\mathrm{C} 8 \mathrm{~A}$ & $0.1998(8)$ & $1 / 4$ & $0.4390(7)$ & $3.5(4)$ \\
$\mathrm{O} 9$ & $0.2805(5)$ & $1 / 4$ & $0.5773(4)$ & $4.7(3)$ \\
$\mathrm{C} 10$ & $0.1769(10)$ & $1 / 4$ & $0.6521(8)$ & $6.0(5)$ \\
$\mathrm{C} 11$ & $0.6806(5)$ & $0.40877(23)$ & $0.4316(4)$ & $2.79(21)$ \\
$\mathrm{C} 12$ & $0.6948(6)$ & $0.4826(3)$ & $0.3960(4)$ & $3.8(3)$ \\
$\mathrm{C} 13$ & $0.7964(6)$ & $0.5306(3)$ & $0.4861(5)$ & $5.3(3)$ \\
$\mathrm{C} 14$ & $0.8851(6)$ & $0.5069(3)$ & $0.6096(5)$ & $5.6(3)$ \\
$\mathrm{C} 15$ & $0.8733(6)$ & $0.4334(3)$ & $0.6468(5)$ & $5.5(3)$ \\
$\mathrm{C} 16$ & $0.7721(6)$ & $0.3844(3)$ & $0.5589(5)$ & $4.1(3)$ \\
$\mathrm{C} 21$ & $0.4410(5)$ & $0.39156(25)$ & $0.1687(4)$ & $3.16(23)$ \\
$\mathrm{C} 22$ & $0.4451(6)$ & $0.3702(3)$ & $0.0460(4)$ & $3.87(25)$ \\
$\mathrm{C} 23$ & $0.3577(7)$ & $0.4082(3)$ & $-0.0680(5)$ & $5.3(3)$ \\
$\mathrm{C} 24$ & $0.2670(7)$ & $0.4678(3)$ & $-0.0585(5)$ & $6.3(3)$ \\
$\mathrm{C} 25$ & $0.2592(7)$ & $0.4892(3)$ & $0.0617(6)$ & $6.4(4)$ \\
$\mathrm{C} 26$ & $0.3456(6)$ & $0.4506(3)$ & $0.1756(5)$ & $4.7(3)$ \\
$\mathrm{C}$ & $0.1812(13)$ & $3 / 4$ & $0.0704(9)$ & $10.5(9)$ \\
$\mathrm{C} 11$ & $0.3691(4)$ & $3 / 4$ & $0.1924(3)$ & $11.7(3)$ \\
$\mathrm{C} 2$ & $0.0418(4)$ & $3 / 4$ & $0.1356(3)$ & $13.2(3)$ \\
\hline
\end{tabular}


Table 6

Some important torsional angles $\left({ }^{\circ}\right.$ ) for complexes $4 \mathrm{~b}, 4 \mathrm{c}$ and 6

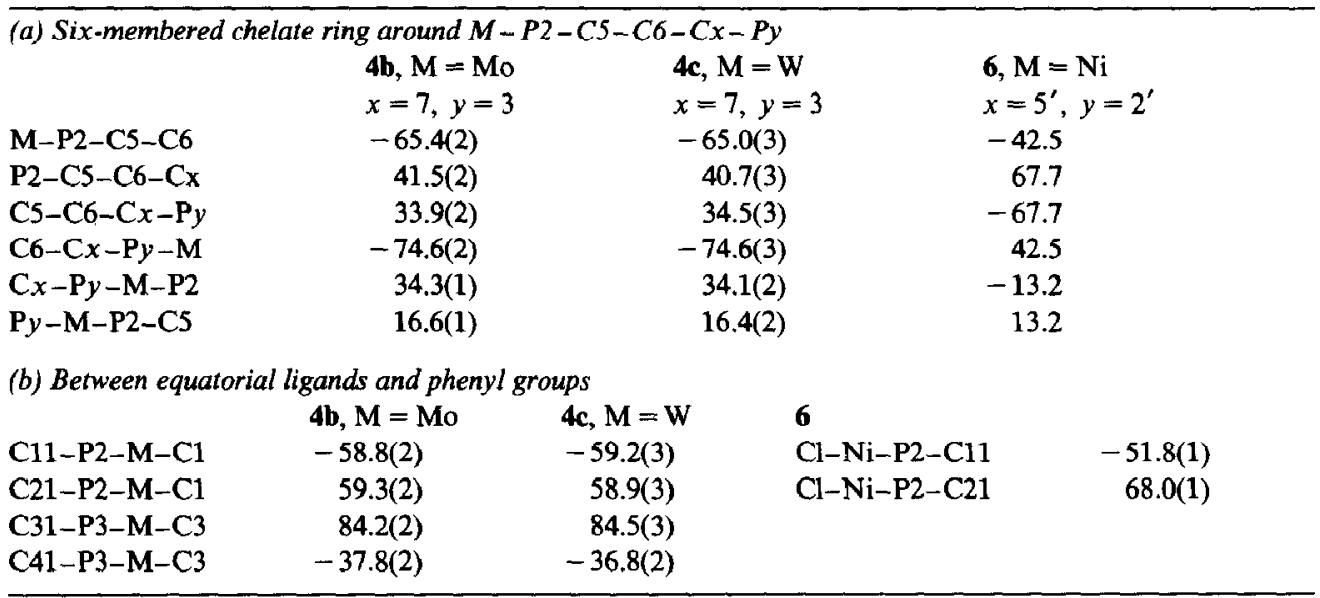

\section{Conformational analysis}

The torsional angles around the six-membered chelate rings for complexes $4 \mathbf{b}, \mathbf{4 c}$ and 6 , are listed in Table 6 for comparison. Species $\mathbf{4 b}$ and $\mathbf{4 c}$ have similar torsional angles around the ring, $\mathrm{M}-\mathrm{P} 2-\mathrm{C} 5-\mathrm{C} 6-\mathrm{C} 7-\mathrm{P3}$, and they share the $+\mathrm{g},-\mathrm{g}$ alternation. The four positive and two negative torsional angles in the rings indicate their chelate rings to be in twist-boat conformations. In contrast, the conformation of the chelate ring on 6 adopts a chair form, which is manifested in the characteristic alternating $+g,-g$ torsional angles. However, this chair conformation is distorted near the P2, Ni, P2' end, as shown in Fig. 4.

Theoretical studies have indicated that the chair conformation is the most stable form in a six-membered chelate ring associated with its octahedral complex [11]; indeed, there are many examples which confirm this. Typically, the structure of (OC) ${ }_{4} \sqrt{\mathrm{PPP}}{ }_{2} \mathrm{CH}\left(\mathrm{CH}_{2} \mathrm{CH}_{2} \mathrm{PPh}_{2}\right) \mathrm{CH}_{2} \mathrm{PPh}_{2}$ (7) shows its chelate ring to be in the chair form with $\mathrm{CH}_{2} \mathrm{PPh}_{2}$ substituent occupying an equatorial position [12]. If $\mathbf{4 b}$ and $\mathbf{4 c}$ were forced into a chair conformation, like in $\mathbf{7}$, three major interactions would arise (Fig. 5): (1) the 1,3-diaxial interaction between methyl and phenyl groups; (2) the "eclipsed" interactions between equatorial ligands and phenyl groups; and (3) the interactions between the apical ligands and hydrogen atoms. Thus, the six-membered chelate rings in $\mathbf{4 b}$ and $\mathbf{4 c}$ adopt a twisted-boat form, which excludes some of those interactions. The dihedral angles between equatorial ligands and phenyl groups (listed in Table 6) in $\mathbf{4 b}$ and $\mathbf{4 c}$ are the result of minimizing the "eclipsed" interactions. The unusually large C31-P3-M-C3 angle makes the C2$\mathrm{M}-\mathrm{P} 3-\mathrm{C} 31\left(\mathrm{M}=\mathrm{Mo},-3.7(2)^{\circ} ; \mathrm{M}=\mathrm{W},-2.9(2)^{\circ}\right)$ angle smaller, and distorts the $\mathrm{P} 3-\mathrm{M}-\mathrm{C} 2$ angle from $90^{\circ}\left[\mathrm{M}=\mathrm{Mo}, 100.4(1)^{\circ} ; \mathrm{M}=\mathrm{W}, 100.7(2)^{\circ}\right]$.

Interestingly, the six-membered chelate ring in species 6 prefers the stable chair form. Without the apical ligands in 6, the interactions between these ligands and hydrogen atoms disappear. The lack of such interaction could allow the chelate ring in 6 to adopt a chair conformation. However, this chair form is distorted (almost into a half-chair form). In order to minimize the "eclipsed" interaction between chlorides and phenyl groups, the dihedral angles of $\mathrm{Cl}-\mathrm{Ni}-\mathrm{P} 2-\mathrm{C} 11$ and $\mathrm{Cl}-\mathrm{Ni}-\mathrm{P} 2-$ 

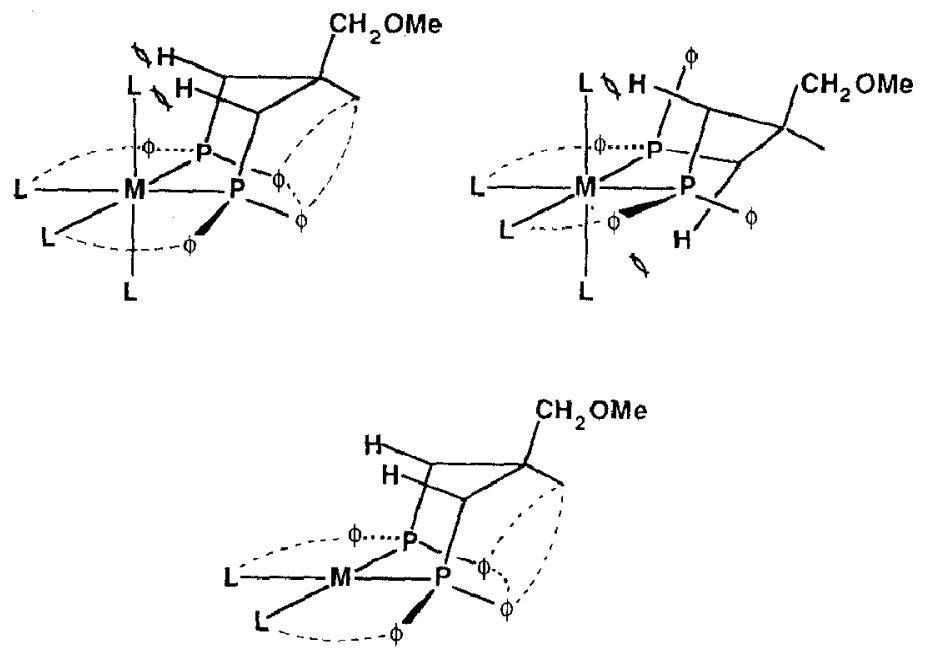

Fig. 5. Major interactions in (a) chair form and (b) twist-boat form in $M\left(P_{2} O\right) \mathbf{L}_{4}$, and (c) chair form in $\mathrm{M}\left(\mathrm{P}_{2} \mathrm{O}\right) \mathrm{L}_{2}$.

$\mathrm{C} 21$ become $-51.8(1)$ and $68.0(1)^{\circ}$ respectively. In addition the larger $\mathrm{P}-\mathrm{Ni}-\mathrm{P}$ $\left[95.49(7)^{\circ}\right]$, angle helps to relieve the 1,3-diaxial interaction between the phenyl groups.

\section{Experimental}

Proton magnetic resonance spectra were recorded on either a Varian EM-390, a Bruker AC-E 200 or a Bruker AM-300WB spectrometer. Proton-decoupled phosphorus-31 NMR spectra were determined on a Bruker AC-E 200 or a Bruker AM-300WB spectrometer at $81.01 \mathrm{MHz}$ and $121.49 \mathrm{MHz}$ respectively. Chemical shifts are given in parts per million relative to $85 \% \mathrm{H}_{3} \mathrm{PO}_{4}$ for ${ }^{31} \mathrm{P}$ NMR spectra in $\mathrm{CDCl}_{3}$, unless otherwise noted.

Infrared and UV/Vis spectra were obtained on a Perkin Elmer 1310 or $983 \mathrm{G}$ and a Perkin Elmer Lambda 3B instrument, respectively. Elemental analyses were carried out on Perkin Elmer $240 \mathrm{C}$ instrument.

All of the reactions, manipulations and purification steps involving phosphines were performed under dry nitrogen or argon. Air-sensitive liquids were transferred by Teflon flex-needles under nitrogen pressure or by syringe. Toluene and xylene were distilled from sodium metal under nitrogen. Acetone was dried over 4A molecular sieves. $\mathrm{NiCl}_{2} \cdot \mathrm{DME}$ was prepared according to the literature method [13]. Other solvents and chemicals from commercial sources were used without further purification, unless otherwise stated.

General procedures for preparation of $4 a-4 c$. A flask equipped with refluxing condenser and containing tripodal ligand $\mathbf{3}$ and equimolar amounts of metal carbonyl in aromatic solvent [toluene for $\mathrm{Mo}(\mathrm{CO})_{6}$ and $\mathrm{W}(\mathrm{CO})_{6}$, xylene for $\left.\mathrm{Cr}(\mathrm{CO})_{6}\right]$, was heated to reflux under nitrogen. The progress of each reaction was monitored by IR spectroscopy in the range of metal-carbonyl absorptions. The crude reaction mixture was then chromatographed on silica gel with a mixture of hexane, dichloromethane and ethyl acetate as eluant. The fraction containing the 
desired product was collected and recrystallized from a solution of methylene chloride and methanol. All spectroscopic and analytical data are listed in Table 1. [2,2-Bis(diphenylphosphinomethyl)-1-methoxypropane]nickel(II) chloride (6). A mixture of 3 (24.3 mg, $0.052 \mathrm{mmol})$ and $\mathrm{NiCl}_{2} \cdot$ DME (11.4 mg, $\left.0.052 \mathrm{mmol}\right)$ in dry acetone $(10 \mathrm{ml})$ was heated at reflux for $2 \mathrm{~h}$. After removal of solvent, the residue was recrystallized from hexane/dichloromethane to give the desired complex 6 as a brown crystalline solid $\left(22.3 \mathrm{mg}, 72 \%\right.$ ): $\mathrm{mp}$ (dec.) $196{ }^{\circ} \mathrm{C} ;{ }^{1} \mathrm{H}$ NMR $\delta 8.10$ (d, $J=7.5 \mathrm{~Hz}, 4 \mathrm{H}), 7.95(\mathrm{~d}, J=7.5 \mathrm{~Hz}, 4 \mathrm{H}), 7.58-7.39(\mathrm{~m}, 12 \mathrm{H}), 3.21-2.56(\mathrm{br}, 4$ $\mathrm{H}), 2.86(\mathrm{~s}, 3 \mathrm{H}), 2.59(\mathrm{~s}, 2 \mathrm{H}), 0.56(\mathrm{~s}, 3 \mathrm{H})$; UV/Vis $\left(\mathrm{CH}_{2} \mathrm{Cl}_{2}\right) 466,312,283,276$, $239 \mathrm{~mm}$.

Anal. Found: $\mathrm{C}$, 54.68; $\mathrm{H}, 5.11 . \mathrm{C}_{30} \mathrm{H}_{32} \mathrm{OP}_{2} \mathrm{Cl}_{2} \mathrm{Ni} \cdot \mathrm{CH}_{2} \mathrm{Cl}_{2}$ calcd.: $\mathrm{C}, 54.30 ; \mathrm{H}$, $5.00 \%$.

[2,2-Bis(diphenylphosphinomethyl)-1-methoxypropane]palladium(II) chloride. A solution of $3(75.5 \mathrm{mg}, 16.06 \mathrm{mmol})$ and $\mathrm{PdCl}_{2}(28.3 \mathrm{mg}, 15.96 \mathrm{mmol})$ in dichloromethane $(2.5 \mathrm{ml})$ and methanol $(2.5 \mathrm{ml})$ was stirred at room temperature overnight. After removal of the solvents, the yellow solid was collected and recrystallized from dichloromethane and methanol. The desired product was isolated as a light yellow solid (45 mg, $43 \%$ ): $\mathrm{mp}$ (dec.) $218^{\circ} \mathrm{C} ;{ }^{1} \mathrm{H}$ NMR $\delta 8.00-7.60$ (m, $8 \mathrm{H}$ ), 7.5-7.4 (m,

Table 7

Crystal data for $4 \mathrm{~b}, \mathbf{4 c}$ and $6^{a . b}$

\begin{tabular}{|c|c|c|c|}
\hline & $\mathbf{4 b}$ & $4 c$ & 6 \\
\hline Formula & $\mathrm{MoP}_{2} \mathrm{C}_{34} \mathrm{H}_{32} \mathrm{O}_{5}$ & $\mathrm{WP}_{2} \mathrm{C}_{34} \mathrm{H}_{32} \mathrm{O}_{5}$ & $\mathrm{NiP}_{2} \mathrm{C}_{31} \mathrm{H}_{34} \mathrm{Cl}_{4} \mathrm{O}$ \\
\hline Crystal size(mm) & $0.30 \times 0.30 \times 0.20$ & $0.50 \times 0.50 \times 0.45$ & $0.2 \times 0.2 \times 0.15$ \\
\hline Lattice & Monoclinic & Monoclinic & Monoclinic \\
\hline Specific gravity & $P 2_{1} / n$ & $P 2_{1} / n$ & $P 2_{1} / m$ \\
\hline$a(\AA ̊)$ & $11.346(4)$ & $11.330(5)$ & $9.173(4)$ \\
\hline$b(\AA)$ & $17.481(3)$ & $17.422(3)$ & $17.726(15)$ \\
\hline$c(\AA)$ & $16.493(4)$ & $16.446(5)$ & $10.693(2)$ \\
\hline$\beta\left({ }^{\circ}\right)$ & $100.97(3)$ & $101.06(3)$ & $110.13(2)$ \\
\hline$V\left(A^{3}\right)$ & $3211(2)$ & $3185(2)$ & $1632(3)$ \\
\hline$Z$ & 4 & 4 & 2 \\
\hline$F(000)$ & 1263.71 & 1363.69 & 1084 \\
\hline Temperature & 300 & 300 & 300 \\
\hline$\mu\left(\mathrm{mm}^{-1}\right)$ & 0.53 & 3.83 & 1.74 \\
\hline Transmission & $0.92-1.00$ & $0.74-1.00$ & $0.90-1.00$ \\
\hline $2 \theta_{\max }\left({ }^{\circ}\right)$ & 49.9 & 49.9 & 50 \\
\hline$h, k, l$ & $13,20, \pm 19$ & $\pm 13,20,19$ & $10,21, \pm 12$ \\
\hline$\lambda(\mathrm{Mo}-K \alpha)(\AA)$ & 0.7093 & 0.7093 & 0.7093 \\
\hline No. of reflections & 5649 & 5599 & 3153 \\
\hline No. of observed reflections & $4439[I>2 \sigma(I)]$ & $3970[I>2 \sigma(I)]$ & $1936[I>2 \sigma(I)]$ \\
\hline No. of variables & 378 & 378 & 190 \\
\hline$R(F)$ & 0.042 & 0.033 & 0.040 \\
\hline$R_{\mathrm{w}}(F)$ & 0.044 & 0.026 & 0.032 \\
\hline$s$ & 4.504 & 2.092 & 1.963 \\
\hline
\end{tabular}

${ }^{a}$ Unit cell parameters were obtained by least-squares refinement of the setting of 24 reflections with $18.25<2 \theta<21.40^{\circ}$ for $4 \mathrm{~b} ; 25$ reflections with $18.66<2 \theta<21.58^{\circ}$ for $4 \mathrm{c}$ and $11.7<2 \theta<32.56^{\circ}$ for 6 .

${ }^{b}$ Relevant expressions are as follows, $F_{0}$ and $F_{c}$ represent respectively the observed and calculated structure factor amplitudes; $R(F)=\Sigma\left|F_{\mathrm{o}}-F_{\mathrm{c}}\right| / \Sigma F_{\mathrm{o}} ; R_{\mathrm{w}}(F)=\left[\sum \mathrm{w}\left(F_{\mathrm{o}}-F_{\mathrm{c}}\right)^{2} / \Sigma \mathrm{w} F_{\mathrm{o}}^{2}\right]^{1 / 2} ; S=\left[\Sigma \mathrm{w}\left(F_{\mathrm{o}}\right.\right.$ $\left.\left.-F_{c}\right)^{2} /(m-n)\right]^{1 / 2}, m=$ No. of reflections, $n=$ No. of variables. 
$12 \mathrm{H}$ ), $2.81(\mathrm{~s}, 3 \mathrm{H}), 2.73(\mathrm{~s}, 2 \mathrm{H}), 2.48$ (dd, $J=15$ and $9 \mathrm{~Hz}, 2 \mathrm{H}$ ), 2.22 (dd, $J=15$ and $9 \mathrm{~Hz}, 2 \mathrm{H}), 0.58(\mathrm{~s}, 3 \mathrm{H}) ;{ }^{31} \mathrm{P}$ NMR $\delta 16.93$; UV/Vis $\left(\mathrm{CH}_{2} \mathrm{Cl}_{2}\right) 320(\epsilon=5300)$, $382(\epsilon=460) \mathrm{nm}$.

Anal. Found: $\mathrm{C}, 52.90 ; \mathrm{H}, 5.08 . \mathrm{C}_{30} \mathrm{H}_{32} \mathrm{OP}_{2} \mathrm{Cl}_{2} \mathrm{Pd} \cdot \frac{1}{2} \mathrm{CH}_{2} \mathrm{Cl}_{2}$ calcd.: $\mathrm{C}, 53.07 ; \mathrm{H}$, $4.82 \%$.

X-Ray data collection. Cell parameters were determined on a CAD-4 diffractometer by a least-squares treatment. Atomic scattering factors were taken from the International Tables for X-Ray Crystallography [14]. The NRCC SDP VAX Program package [15] was used. Relevant data are listed in Table 7. Tables of atomic coordinates and thermal parameters, anisotropic thermal parameters, complete bond distances and bond angles, and structure factors are available from the authors.

\section{Acknowledgement}

Financial support of this work by the National Science Council (NSC77-0208M002-49) is acknowledged.

\section{References}

1 For recent review on tripodal polytertiary phosphines see: (a) L. Sacconi and F. Mani, in G.A. Melson and B.N. Figgis (Eds.), Transition Metal Chemistry, Vol. 8, Marcel Dekker, New York, 1982, p. 179, (b) D.W. Meek, in L.H. Pignolet (Ed.), Homogeneous Catalysis by Metal Phosphine Complexes, Plenum, New York, 1983, p. 257.

2 (a) A. Albinati, F. Demartin, P. Janser, L.F. Rhodes, L.M. Venanzi, J. Am. Chem. Soc., 111 (1989) 2115; N. Kuhn, M. Winter, E. Zimmer, J. Organomet. Chem., 344 (1988) 401; J.R. Bleeke, M.K. Hays, R.J. Wittenbrink, Organometallics, 7 (1988) 1417; G.V. Goeden, J.C. Hoffman, K.G. Caulton, Inorg. Chem., 25 (1986) 2484; M.D. Vaira, M. Peruzzini, P. Stoppioni, Polyhedron, 5 (1986) 945; P. Peringer, M. Lusser, Inorg. Chim. Acta, 117 (1986) L25; S.C. Abrahams, A.P. Ginsberg, T.F. Koetzle, P. Marsh, C.R. Sprinkle, Inorg. Chem., 25 (1986) 2500: S.I. Hommeltoft, A.D. Cameron. T.A. Shackleton, M.E. Fraser, S. Fortier, M. C. Baird, Organometallics, 5 (1986). 1380; O.J. Scherer, R. Walter, W.S. Sheldrick, Angew. Chem. Int. Ed. Engl., 24 (1985) 525; F. Cecconi, C.A. Ghilardi, S. Midollini, S. Moneti, A. Orlandini, M. Bacci, J, Chem. Soc., Chem. Commun., (1985) 731; J. Rimmelin, P. Lemoine, M. Gross, A.A. Bahsoun, J.A. Osborn, Nouv. J. Chim., 9 (1985) 181; D.J. Darensbourg, D.J. Zalewski, Organometallics, 4 (1985) 92; R.B. King, P.N. Kapoor, R.N. Kapoor, Inorg. Chem., 10 (1971) 1841; J. Chatt, F.A. Hart, H.R. Watson, J. Chem. Soc., (1962) 2537, and references therein.

3 A.A. Ismail, I.S. Butler, J. Organomet. Chem., 346 (1988) 185.

4 C. Bianchini, D. Masi, D. Mealli, A. Meli, M. Sabat, F. Vizza, Inorg. Chem., 27 (1988) 3716; C. Bianchini, F. Laschi, A. Meli, M. Peruzzini, P. Zanello, P. Frediani, Organometallics, 7 (1988) 2575; C. Bianchini, D. Masi, A. Meli, M. Peruzzini, F. Zanobini, J. Am. Chem. Soc., 110 (1988) 6411, and references therein.

5 R. Davis, J.E. Fergusson, Inorg. Chim. Acta, 4 (1970) 23.

6 S.-T. Liu, C.-H. Yieh, H.-J. Lu, Phosphorus, Sulfur and Silicon, 44 (1989) 261.

7 S.-T. Liu, H.-E. Wang, M.-C. Cheng, S.-M. Peng, J. Organomet. Chem., 376 (1989) 333.

8 A.J. McAlees, R. McCrindle, A.R. Woon-Fat, Inorg. Chem. 15 (1976) 1065.

9 P.E. Garrou, Chem. Rev., 81 (1981) 229.

10 D. Berglund, Ph.D. dissertation, Ohio State University, 1969; also see reference 1a.

11 J.R. Gollogly, C.J. Hawkins, Inorg. Chem., 11 (1972) 156.

12 M.R. Churchill, A.L. Rheingold, R.L. Keiter, Inorg. Chem., 20 (1981) 2730.

13 L.G.L. Ward, Inorg. Synth., 13 (1972) 154.

14 International Tables for X-Ray Crystallography. Vol. IV, Kynoch Press, Birmingham, 1974.

15 E.J. Gabe, F.L. Lee, Acta Crystallogr., A37 (1981) S 339. 